\title{
iASPP overexpression is associated with clinical outcome in spinal chordoma and influences cellular proliferation, invasion, and sensitivity to cisplatin in vitro
}

\author{
Yunlong Ma ${ }^{1, *}$, Bin Zhu ${ }^{2, *}$, Xiaoguang Liu' ${ }^{1}$, Zhongjun Liu ${ }^{1}$, Liang Jiang ${ }^{1}$, Feng Wei ${ }^{1}$, \\ Miao $Y u^{1}$, Fengliang $W_{u^{1}}$, Hua Zhou ${ }^{1}$, Nanfang $X u^{1}$, Xiao Liu ${ }^{1}$, Lei Yong ${ }^{1}$, Yongqiang \\ Wang $^{1}$, Peng Wang ${ }^{1}$, Chen Liang ${ }^{1}$ and Guanping $\mathbf{H e}^{1}$ \\ ${ }^{1}$ Department of Orthopedics, Peking University Third Hospital, Beijing 100191, China \\ ${ }^{2}$ The Center for Pain Medicine, Peking University Third Hospital, Beijing 100191, China \\ *Authors contributed equally to this work \\ Correspondence to: Xiaoguang Liu, email: xgliudoctor@163.com
}

Keywords: chordoma, iASPP, prognosis, invasion, cisplatin

Received: November 30, $2016 \quad$ Accepted: August 06, 2017 Published: August 11, 2017

Copyright: Ma et al. This is an open-access article distributed under the terms of the Creative Commons Attribution License 3.0 (CC BY 3.0 ), which permits unrestricted use, distribution, and reproduction in any medium, provided the original author and source are credited.

\section{ABSTRACT}

The oncogenetic function of inhibitory member of the apoptosis stimulating protein of p53 family (iASPP) in chordoma is unclear and remains to elucidate. The expression of iASPP in chordoma tissues and cells, its correlation to clinicopathological parameters and the effect on the patients' prognosis were evaluated. Cellular proliferation, invasion and cisplatin-response were observed after the iASPP knockdown or overexpression in vitro. Co-Immunoprecipitation assay was used to explore the interaction between iASPP and p53. The regulation of miRNA-124 on the expression and apoptotic function of iASPP was explored after transiently transfecting cells with miRNA-124 mimics or inhibitor. Results indicated that iASPP overexpressed in chordoma tissues and cells. Its overexpression was associated with tumor invasion and local recurrence, and was predictive of patients' poor prognosis. Cells with iASPPsilence showed a decreased ability of proliferation and invasion, but an increasing sensitivity to cisplatin. Besides, iASPP could combine with p53 in either endogenous or exogenous detection. Post-transcriptionally, miRNA-124 negatively regulated the expression of iASPP, which further led to the changes of apoptosis-related proteins. Thus, iASPP overexpression is associated with the clinical outcome in spinal chordoma and influences cellular proliferation, invasion, and the sensitivity to cisplatin.

\section{INTRODUCTION}

Chordoma is a primary malignant bone tumor originated from primitive notochord remnants with strong local invasiveness and a high recurrence rate. It accounts for $1 \sim 4 \%$ of all primary malignant skeletal tumors and the overall incidence is relatively low [1,2]. The 5- and 10 -year survival rates of the patients are approximately $68 \%$ and $40 \%$, respectively [3]. Standard treatment of chordoma consists of surgery and radiotherapy due to its resistance to chemotherapeutics $[4,5]$. Even the patients received extensive surgical resection, local recurrence is still common $[6,7]$. Although the excellent response to radiotherapy reported, the clinical application and treatment efficacy of this adjuvant therapy are still limited and debated $[8,9]$. Given this, the identification of novel biomarkers could be helpful for the prediction, diagnosis and precise target therapy for chordoma.

The dysfunction of p53 is an important process in tumorigenesis, and mainly results from p53 mutation or inactivation [10]. Although p53 dysfunction is associated with chordoma tumorigenesis $[11,12]$, genetic alternation of the encoding gene appears to be an uncommon event as mutations in specific exons of $\mathrm{p} 53$ have not been observed [11]. In addition, although the loss of heterozygosity on chromosome $17 \mathrm{p} 13$, where $\mathrm{p} 53$ located, has been reported 
in some chordoma cases, this event is not correlated to abnormal p53 expression or overall survival rate [12]. These findings suggest that mutation may be not the primary cause of p53 dysfunction in chordoma.

iASPP, the inhibitory member of the apoptosis stimulating protein of p53 (ASPP) family, is encoded by PPP1R13L gene [13]. It was initially identified as Relassociated inhibitor (RAI), as its interaction with nuclear factor kappa B (NF-кB) subunit p65 (RelA) suppresses its transcriptional activity [14]. iASPP is the exclusive, evolutionarily conserved p53 suppressor in this family, whereas another two members, namely ASPP1 and ASPP2 are p53 activators [14, 15]. iASPP has been reported to overexpress in many human tumors, including non-small cell lung cancer, breast cancer, hepatocellular carcinoma, cervical cancer, and leukemia [16-20]. Its overexpression confers proliferative, migratory, and invasive capacity to cancer cells, and is associated with tumor recurrence, chemotherapy tolerance, and poor prognosis [16, 19, 21-23]. Inhibition of iASPP expression or disruption of iASPP-p53 interaction in tumor cells induces apoptosis and growth suppression in tumor cells, and even enhances chemotherapy sensitivity [24, $25]$. Recently, growing studies have reported the cancerrelated roles of microRNAs (miRNAs) [26, 27] and their regulation on iASPP expression [28, 29]. Altered expression of iASPP in many tumor cells can be posttranscriptionally regulated by various miRNAs such as miRNA-124 [30-33], miRNA-140 [34, 35], miRNA-184 [36] and miRNA-506 [37], which involve in modulating the growth or invasion of these cells by targeting iASPP. Therein, studies have well documented that the 3 '-UTR of iASPP includes the binding sites of miRNA-124, indicating the important role of miRNA-124 in the regulation of iASPP.

To our knowledge, no study has thus far reported the function of iASPP in chordoma. In view of the strong local invasion and chemotherapy tolerance of chordoma as well as the important oncogenetic function of iASPP reported in other tumors, iASPP expression in chordoma and its exact regulation on the cellular proliferation, invasiveness, and cisplatin response were observed in this study. What's more, the potential regulation of miRNA-124 on apoptosis function of iASPP was also explored.

\section{RESULTS}

\section{Characterization of tumor tissues as conventional-type chordomas}

The results of hematoxylin and eosin (HE) staining showed that all samples presented a classic lobular architecture separated by fibrous septa. Sheets or elongated cords of clear cells with multiple intracytoplasmic vacuoles distributed in tumor nests with abundant eosinophilic cytoplasm and round nuclei within myxoid matrices, which is the pathognomonic feature of conventional-type chordoma (Supplementary Figure 1A). In addition, these samples were identified based on immunohistochemistry (IHC), staining for diagnostic biomarkers of chordoma including cytokeratin, vimentin, EMA, S-100, and brachyury (Supplementary Figure 1B-1F).

\section{iASPP is overexpressed in chordoma tissues and cells}

IHC showed that iASPP predominantly expressed in the cytoplasm of chordoma cells with diverse intensities (Supplementary Figure 2A-2D). iASPP expression in chordoma tissues was elevated (Figure 1A) when compared to that in distant normal tissues (Figure 1B) and nucleus pulposus (NP) (Figure 1C) with a statistically significant difference $(p=0.000$, iASPP expression in chordoma vs. distant normal tissues; $p=0.009$, iASPP expression in chordoma vs. NP) (Table 1). Consistently, iASPP overexpression in chordoma tissues and cells was also confirmed by either real-time quantitative polymerase chain reaction (RT-qPCR) (Figure 1D, 1E) or western blotting (WB) (Figure 1F, 1G).

\section{iASPP overexpression correlates to tissue invasion, tumor recurrence, and poor prognosis}

Pearson's Chi-square analysis indicated that high iASPP expression was not statistically associated with patient age $(p=0.214)$, gender $(p=0.445)$, tumor location $(p=0.919)$, or tumor size $(p=0.935)$ (Table 2). However, samples from patients that presented surrounding tissue invasion exhibited increased iASPP positivity $(88.9 \%, 16 / 18)$ compared to that in samples from patients without surrounding invasion $(38.5 \%, 5 / 13)$, and this difference was statistically significant ( $p=0.003$; Table 2). During follow-up, 18 patients experienced local recurrence and the median recurrence time was $20.1 \pm 14.8$ months. Based on all samples with high iASPP expression, 16 of 21 patients $(76.2 \%)$ developed recurrence, whereas in samples with low iASPP expression, only 2 of 10 patients $(20.0 \%)$ experienced recurrence, and this difference was statistically significant $(p=0.003)$. At the end of followup, tumor-related death occurred in 11 patients, including two cases of low iASPP expression and nine cases of high iASPP expression. Furthermore, a Kaplan-Meier survival plot and a log-rank test also suggested that the continuous disease-free survival time (CDFS) of patients with high iASPP expression was much shorter than that in patients with low iASPP expression ( $p=0.001$; Figure 2A). Similarly, the cumulative total survival rate in the high expression group was notably lower than that in the low expression group ( $p=0.023$; Figure $2 \mathrm{~B})$. 
Table 1: Expression of iASPP in chordoma, distant normal tissues and nucleus pulposus samples

\begin{tabular}{ccccc}
\hline \multirow{2}{*}{ Tissue sample } & \multirow{2}{*}{} & \multicolumn{2}{c}{ iASPP expression } & \multirow{2}{*}{ H value } \\
\cline { 3 - 3 } & & High (\%) & Low (\%) & \\
\hline Chordoma tissues & 31 & $21(67.7 \%)$ & $10(32.3 \%)$ & \\
Distant normal tissue & 18 & $2(11.1 \%)$ & $16(88.9 \%)$ & $0.000^{*}$ \\
Nucleus pulposus & 15 & $4(26.7 \%)$ & $11(73.3 \%)$ & $0.009^{*}$ \\
\hline
\end{tabular}

\section{Cellular proliferation is decreased by} iASPP knockdown but increased by iASPP overexpression

In order to explore the potential function of iASPP gene in chordoma, we established the knockdown and overexpression systems of iASPP by transfecting both cells with pLKD-iASPP-shRNAs and pLentiEGFP-iASPP. The results of WB assay confirmed that endogenous iASPP successfully silenced by pLKDiASPP-shRNAs and overexpressed by pLenti-EGFPiASPP (Figure 3A).

The effect of iASPP overexpression and knockdown on cellular proliferation was determined using CCK- 8 and colony formation assays. CCK-8 assay (Figure 3B) demonstrated that when compared to that in the pLKDshRNA-Control group, the proliferation of MUG-Chor1 cells harboring pLKD-iASPP-shRNA1 and pLKD-
iASPP-shRNA2 was remarkably reduced after culturing for $4 \mathrm{~d}$ (0.79-fold, $p<0.001$ and 0.72-fold, $p<0.001$, respectively) and $7 \mathrm{~d}$ (0.94-fold, $p<0.05$ and 0.87 fold, $p<0.01$, respectively). However, the proliferation of MUG-Chor1 cells expressing pLenti-EGFP-iASPP was notably increased after culture for $4 \mathrm{~d}$ (1.34-fold, $p<0.001)$ and $7 \mathrm{~d}(1.11$-fold, $p<0.01)$ when compared to that of the pLenti-EGFP-Control group. Similarly, the proliferation of U-CH1 cells with pLKD-iASPP-shRNA1 and pLKD-iASPP-shRNA2 also obviously reduced after culturing for $4 \mathrm{~d}$ (0.85-fold, $p<0.01$ and 0.79 -fold, $p<0.001$, respectively) and $7 \mathrm{~d}(0.89$-fold, $p<0.001$ and 0.93 -fold, $p<0.01$, respectively). In contrast, the proliferation of U-CH1 cells harboring pLenti-EGFPiASPP prominently increased after culturing for $4 \mathrm{~d}(1.18$ fold, $p<0.001)$ and $7 \mathrm{~d}(1.12$-fold, $p<0.01)$.

Accordingly, colony formation in the two iASPPsilenced groups was lower than that in the corresponding
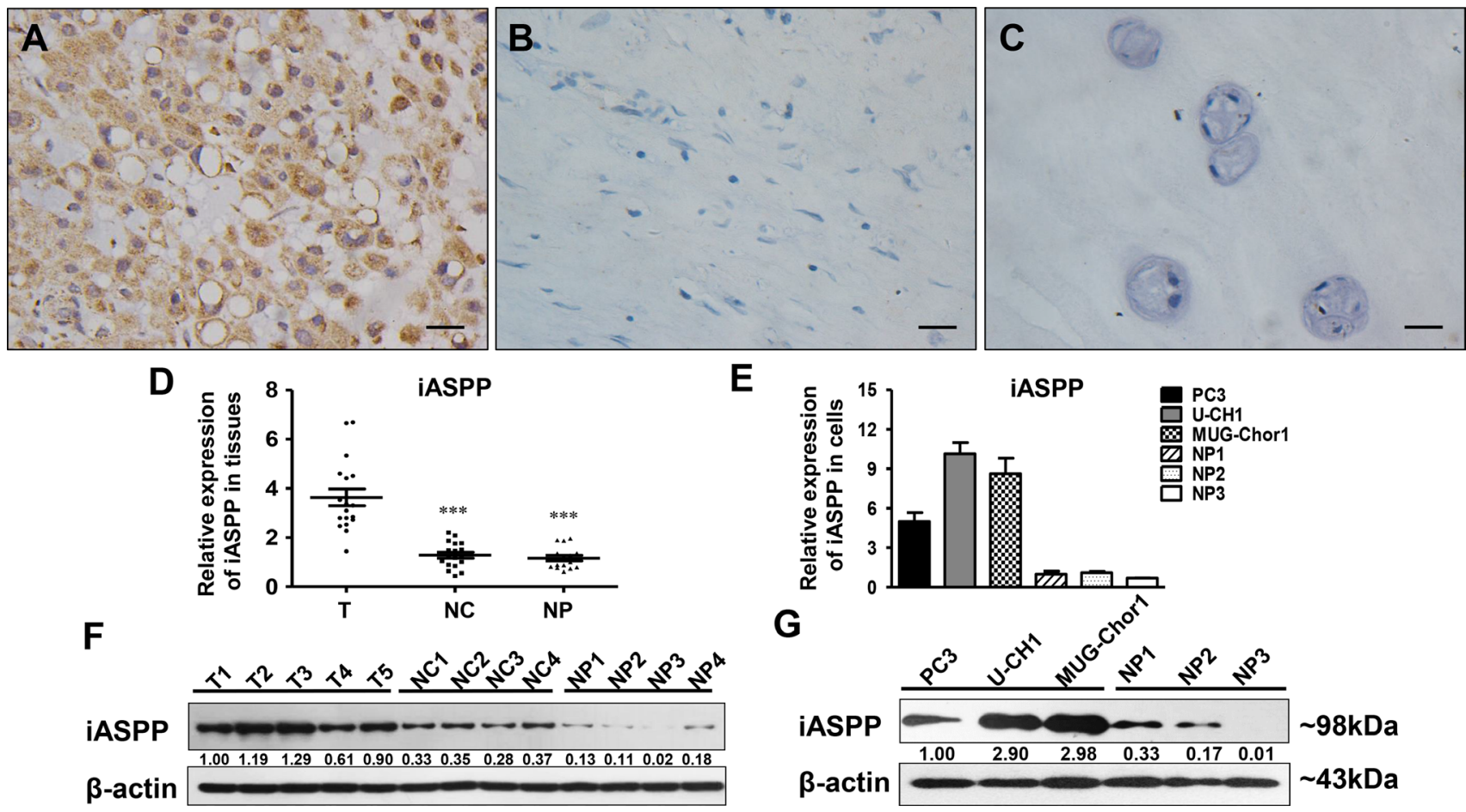

E

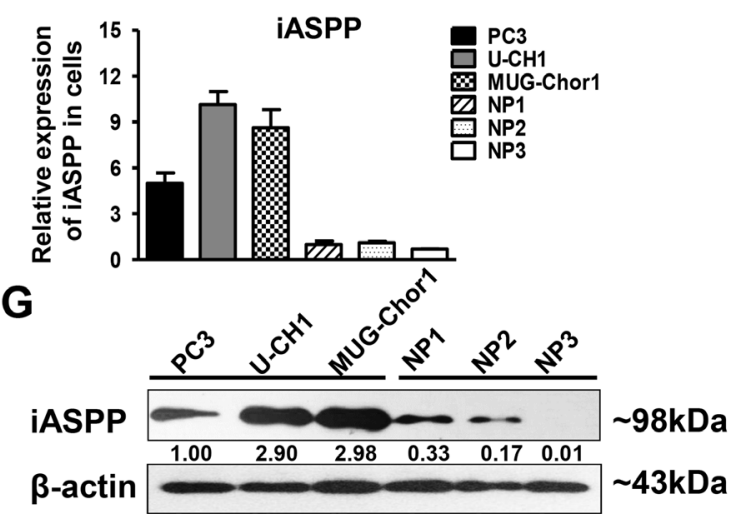

Figure 1: iASPP expression in chordoma tissues and cells. (A-C) Immunohistochemistry staining of iASPP in chordoma tissues (A), distant normal control tissues (B), and nucleus pulposus (C). T: tumor group, NC: normal control tissues group, NP: nucleus pulposus tissues group. (D) Relative expression of iASPP in tissues as detected by RT-qPCR. ${ }^{* * *} p<0.001 v s$. tumor group. (E) Relative expression of iASPP in cells as detected by RT-qPCR. (F) Relative expression of iASPP in tissues as detected by WB. (G) Relative expression of iASPP in cells as detected by WB. Numbers below pictures of F and G represent semi-quantitative analysis of each line by Quantity One software. Bar scale $=20 \mu \mathrm{m}$. 
Table 2: Association of iASPP expression with clinical parameters in conventional chordoma

\begin{tabular}{|c|c|c|c|c|}
\hline \multirow{2}{*}{ Parameters } & \multirow{2}{*}{$N$} & \multicolumn{2}{|c|}{ iASPP expression } & \multirow{2}{*}{$p$ value } \\
\hline & & High & Low & \\
\hline \multicolumn{5}{|l|}{ Age (years) } \\
\hline$<50$ & 11 & 9 & 2 & \multirow{2}{*}{0.214} \\
\hline$\geq 50$ & 20 & 12 & 8 & \\
\hline \multicolumn{5}{|l|}{ Gender } \\
\hline Male & 22 & 14 & 8 & \multirow{2}{*}{0.445} \\
\hline Female & 9 & 7 & 2 & \\
\hline \multicolumn{5}{|l|}{ Tumor Location } \\
\hline Cervical & 19 & 13 & 6 & \multirow{2}{*}{0.919} \\
\hline Sacrococcygeal & 12 & 8 & 4 & \\
\hline \multicolumn{5}{|l|}{ Tumor size $(\mathrm{mm})$} \\
\hline$<90$ & 22 & 15 & 7 & \multirow{2}{*}{0.935} \\
\hline$\geq 90$ & 9 & 6 & 3 & \\
\hline \multicolumn{5}{|c|}{ Surrounding tissue invasion } \\
\hline Yes & 18 & 16 & 2 & \multirow{2}{*}{$0.003^{*}$} \\
\hline No & 13 & 5 & 8 & \\
\hline \multicolumn{5}{|l|}{ Recurrence } \\
\hline Yes & 18 & 16 & 2 & \multirow[t]{2}{*}{$0.003^{*}$} \\
\hline No & 13 & 5 & 8 & \\
\hline
\end{tabular}

control group (Figure 4A). The corresponding fold change was about 0.45 -fold in the iASPP-shRNA1 group $(p<0.001)$ and 0.42 -fold in the iASPP-shRNA2 $(p<0.001)$ for MUG-Chor1, and 0.54-fold in the iASPP-shRNA1 group $(p<0.001)$ and 0.59 -fold in the iASPP-shRNA2 $(p<0.001)$ for U-CH1 (Figure 4B). However, the number of colonies formed in the iASPPoverexpressing group was dramatically higher than that in the corresponding control group (Figure 4C), which was approximately 2.00 -fold for MUG-Chor $1(p<0.001)$ and 1.97 -fold for U-CH1 $(p<0.001)$ (Figure 4D).

\section{Cells invasion is suppressed by iASPP knockdown but induced by iASPP overexpression}

Results of transwell invasion assays showed that the number of invading cells was significantly reduced with iASPP downregulation in both chordoma cell lines in comparison with the corresponding control group (Figure 5A). The corresponding fold change is about 0.50 fold in the iASPP-shRNA1 group $(p<0.001)$ and 0.41 -fold in the iASPP-shRNA2 $(p<0.001)$ for MUG-Chor1; and 0.31fold in the iASPP-shRNA1 group $(p<0.001)$ and 0.38 -fold in the iASPP-shRNA2 $(p<0.001)$ for U-CH1 (Figure 5B). However, iASPP overexpression remarkably promoted the invasion of these chordoma cell lines (Figure 5C). The fold change is approximately 1.13 -fold $(p<0.05)$ for MUGChor1 and 1.34-fold $(p<0.01)$ for U-CH1 (Figure 5D).

\section{Cells sensitivity to cisplatin is enhanced by iASPP knockdown but attenuated by iASPP overexpression}

After treatment with $20 \mu \mathrm{M}$ cisplatin for $48 \mathrm{~h}$, the apoptosis of both MUG-Chor1 and U-CH1 cells was obviously elevated upon iASPP silencing when compared to that in the controls (Figure 6A). For MUG-Chor1 cells, the apoptosis rate was about $50.06 \%$ in the iASPPshRNA1 group $(p<0.05)$ and $50.84 \%$ in the iASPPshRNA2 group $(p<0.05) v s .30 .22 \%$ in the shRNA control group (Figure 6B). For U-CH1 cells, the rate was about $53.74 \%$ in the iASPP-shRNA1 group $(p<0.001)$ and $54.50 \%$ in the iASPP-shRNA2 group $(p<0.001)$ vs. $30.42 \%$ in the shRNA control group (Figure 6B). In contrast, the apoptosis of both cells was reduced with iASPP overexpression when compared to that in the corresponding controls (Figure 7A). The apoptosis rate was approximately $23.97 \%$ in the EGFP-iASPP group vs. $42.78 \%$ in the EGFP-control group for MUG-Chor 1 $(p<0.01)$, and $21.05 \%$ in the EGFP-iASPP group vs. $37.40 \%$ in the EGFP-control group for U-CH1 $(p<0.01)$ (Figure 7B).

\section{miRNA-124 down-regulates iASPP expression}

The expression of miRNA-124 in patients' tissue samples was firstly determined by RT-qPCR. The results indicated that the level of miRNA-124 in the tumor group 
A

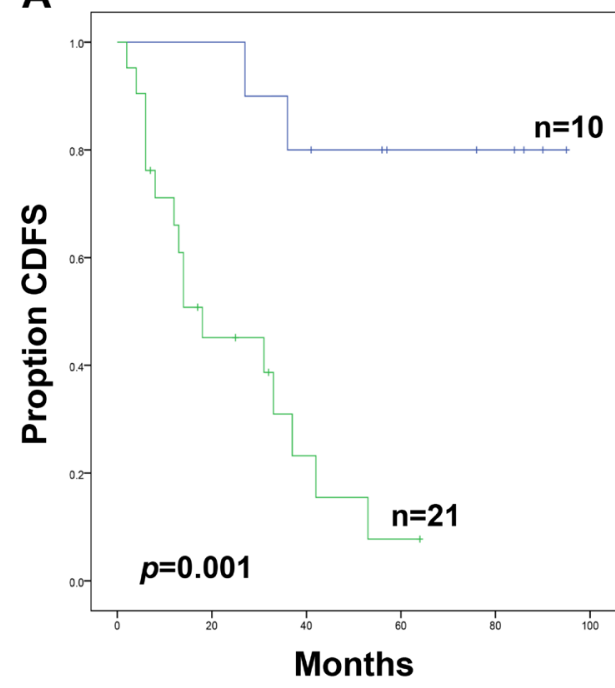

B

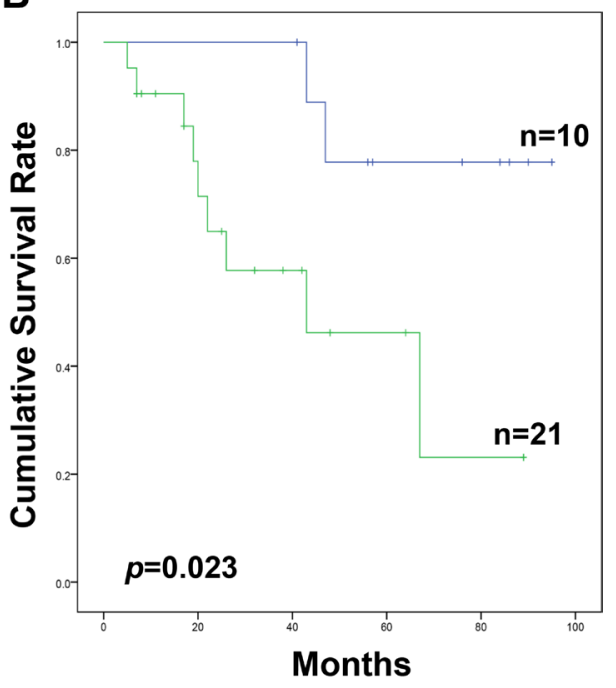

CDFS: Continuous disease-free survival time

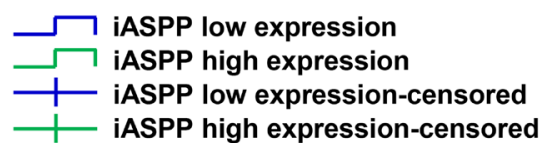

Figure 2: The association of iASPP expression with the prognosis of patients with spinal chordoma. (A and B) KaplanMeier survival analysis and log-rank test of continuous disease-free survival (A) and cumulative survival rate (B).

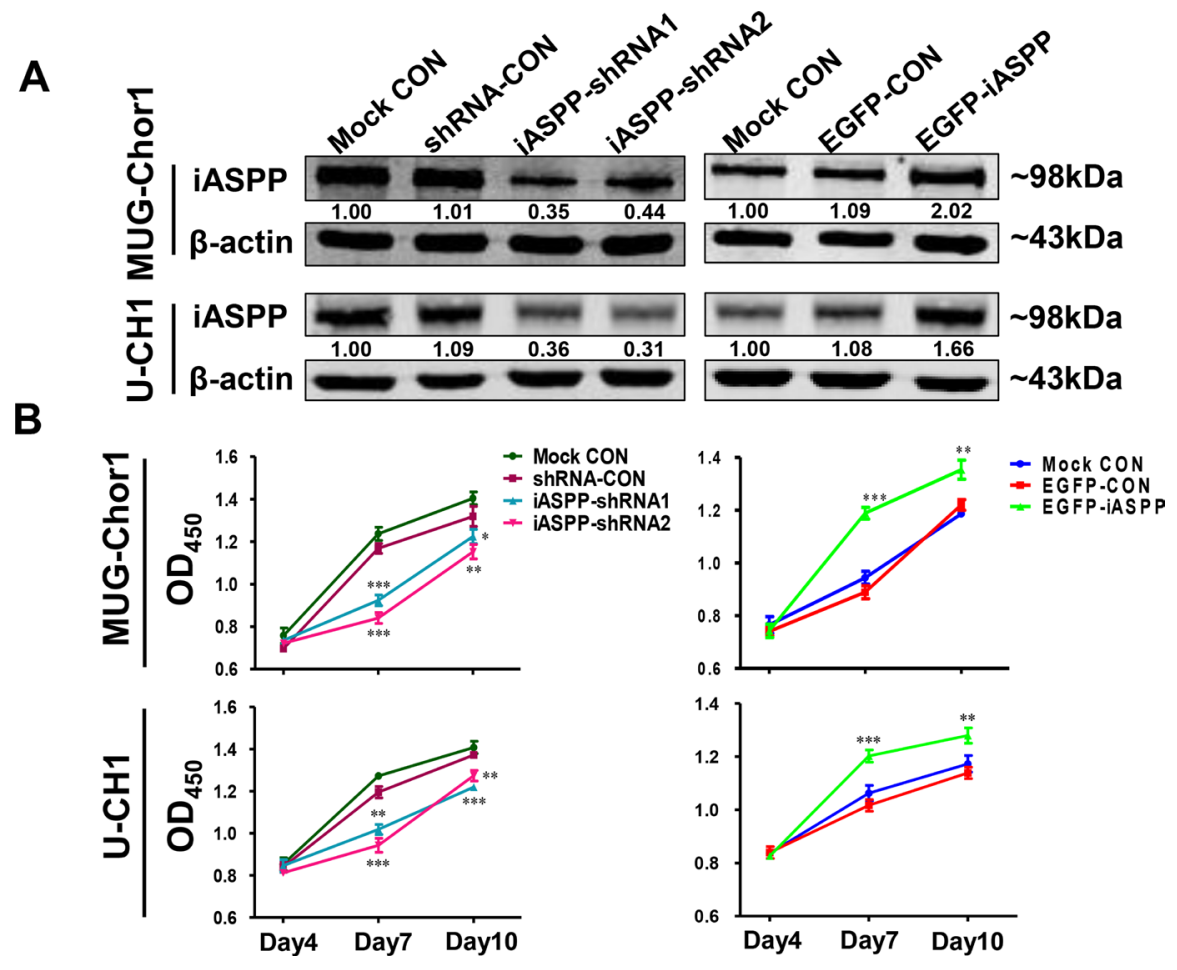

Figure 3: The extrinsic knockdown and overexpression of iASPP and their effects on cells proliferation based on CCK8 assays. (A) The extrinsic knockdown and overexpression of iASPP in MUG-Chor1 and U-CH1 cells as identified by WB. Numbers below pictures represent semi-quantitative analysis of each line by Quantity One software. (B) Proliferation of both cells with iASPP knockdown and overexpression. $n=3$. Mean \pm SEM. ${ }^{*} p<0.05,{ }^{* *} p<0.01,{ }^{* * *} p<0.001$, iASPP-shRNA1 group or iASPP-shRNA2 group vs. shRNA-CON group, and EGFP-iASPP group vs. EGFP-CON group. CON: control. 


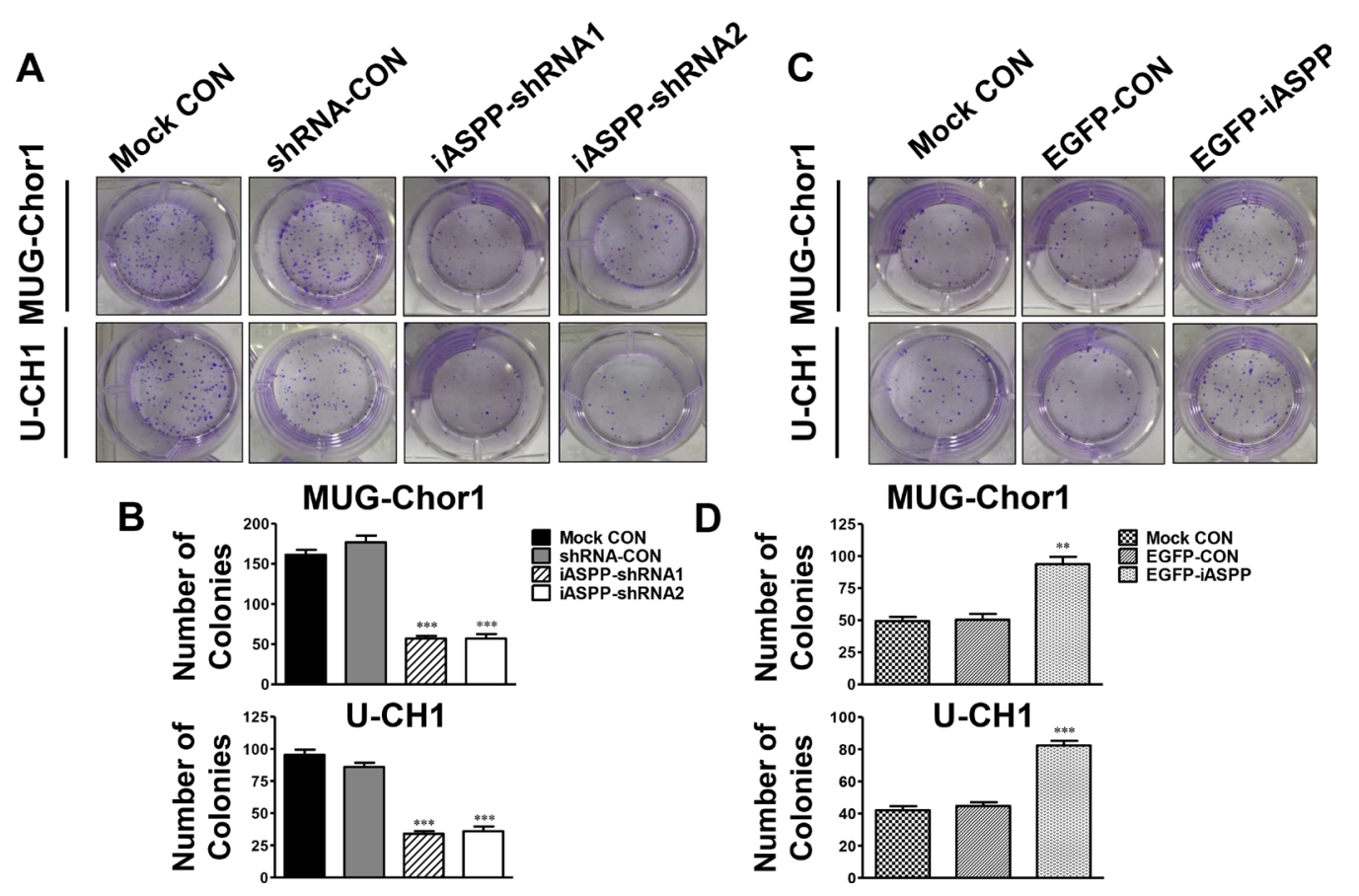

Figure 4: Effect of iASPP knockdown and overexpression on colony formation. (A) Pictures of colony formation assay in MUG-Chor1 and U-CH1 cells after the iASPP knockdown with iASPP-shRNAs. (B) The number of colonies in both cells after the iASPP knockdown with iASPP-shRNAs. (C) Pictures of colony formation assay in MUG-Chor1 and U-CH1 cells after the iASPP overexpression with EGFP-iASPP. (D) The number of colonies in both cells after the iASPP overexpression with EGFP-iASPP. $n=3$. Mean \pm SEM. ${ }^{* *} p<0.01,{ }^{* * *} p<0.001$, iASPP-shRNA1 group or iASPP-shRNA2 group $v s$. shRNA-CON group, and EGFP-iASPP group $v s$. EGFP-CON group. CON: control.

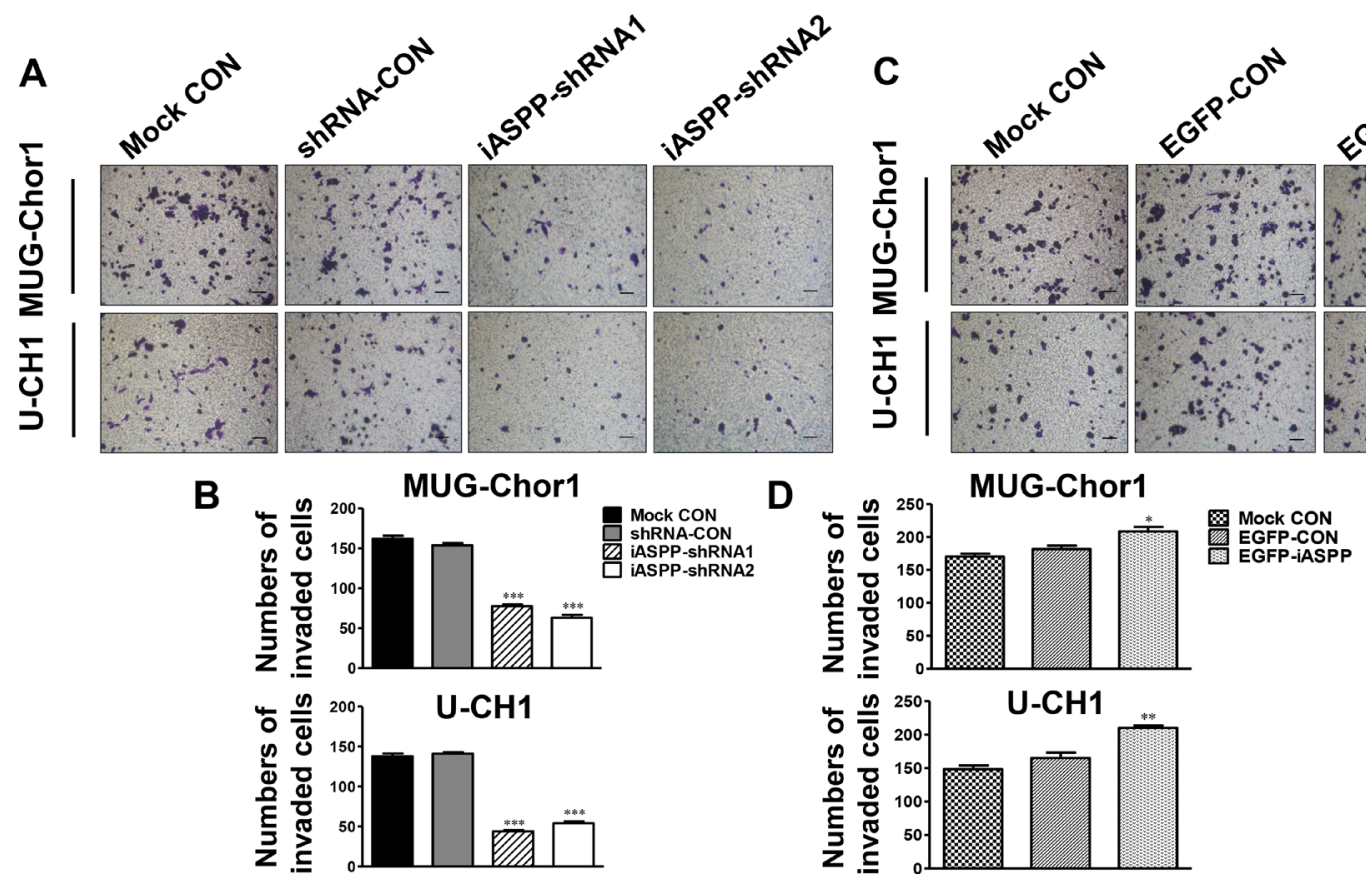

Figure 5: Effect of iASPP knockdown and overexpression on cells invasion. (A) Pictures of transwell invasion assays in MUGChor1 and U-CH1 cells after the iASPP knockdown with iASPP-shRNAs. (B) The number of both cells that invaded the substratum of the membrane per view under a $100 \times$ magnification after the iASPP knockdown with iASPP-shRNAs. (C) Pictures of transwell invasion assays in MUG-Chor1 and U-CH1 cells after the iASPP overexpression with EGFP-iASPP. (D) The number of both cells that invaded the substratum of the membrane per view under a $100 \times$ magnification after the iASPP overexpression with EGFP-iASPP. $n=3$. Mean \pm SEM. ${ }^{*} p<0.05,{ }^{* *} p<0.01,{ }^{* * *} p<0.001$, iASPP-shRNA1 group or iASPP-shRNA2 group $v s$. shRNA-CON group, and EGFP-iASPP group $v s$. EGFP-CON group. CON: control. Bar scale $=100 \mu \mathrm{m}$. 
was significantly lower than that in the control groups that including distant normal tissues $(p<0.001)$ and NP tissues $(p<0.001)$ (Figure 8A). Subsequently, we further observed the regulation of miRNA-124 on iASPP expression in both MUG-Chor1 and U-CH1 cells. As shown in Figure 8B, miRNA-124 presented a negative regulation on iASPP expression at mRNA level in both cells after respectively transfecting cells with miRNA-124 mimics and inhibitor. iASPP was significantly downregulated in cells transfected with miRNA-124 mimics but slightly up-regulated with miRNA-124 inhibitor. What's more, the same findings were also observed at protein level (Figure 8C). All these results suggested that the down-regulation of miRNA-124 existed in chordoma cells and its dysregulation inversely regulated the expression of iASPP.

\section{miRNA-124 inversely regulates the apoptosis function of iASPP}

The co-immunoprecipitation for exogenous detection was performed in HEK293 cells that cotransfected with pCMV-FLAG-p53 and pCMV-HAiASPP for 48 h. Figure 9A demonstrated that FLAG-p53 could interact with iASPP in exogenous detection. In order to observe the endogenous interaction between iASPP and p53, cell lysates of MUG-Chor1 and U-CH1 were immunoprecipitated with anti-p53 antibody and immunoblotted with anti-iASPP antibody. Figure 9B showed that p53 also interacted with iASPP in endogenous detection. All these data confirmed that the interaction between iASPP and p53 existed in chordoma cells.

By transfecting both chordoma cells with iASPP, iASPP + miRNA-124 mimics and iASPP + miRNA-NC, we also evaluated the potential effect of miRNA-124 targeted to iASPP on certain apoptosis-related proteins including $\mathrm{p} 53$, bax, bcl-2, and caspase-3. The results indicated that iASPP significantly inhibited p53 expression and up-regulated anti-apoptotic protein bcl-2, but obviously down-regulated pro-apoptotic proteins bax and caspase-3. However, the inhibition of iASPP by miRNA-124 mimics presented the reverse regulation effects on these proteins (Figure 9C).

\section{DISCUSSION}

Chordoma mainly involves the axial skeleton with strong local invasiveness and a high recurrence rate. It is an intractable disease as even with complete resection, local recurrence is still common. Researchers have demonstrated that p53 dysfunction is one of the causes in tumorigenesis of chordoma; however, p53 mutation is not a common event in this disease $[11,38]$. Evidence suggests that there are several other factors interrupt the normal function of p53. iASPP, the most evolutionarily conserved member in ASPP family, can inhibit apoptosis by specifically interfering with the transactivation of p53-family members to the promoters of apoptosisrelated genes [14, 39]. Thus far, the abnormal expression of iASPP has been widely reported in many types of
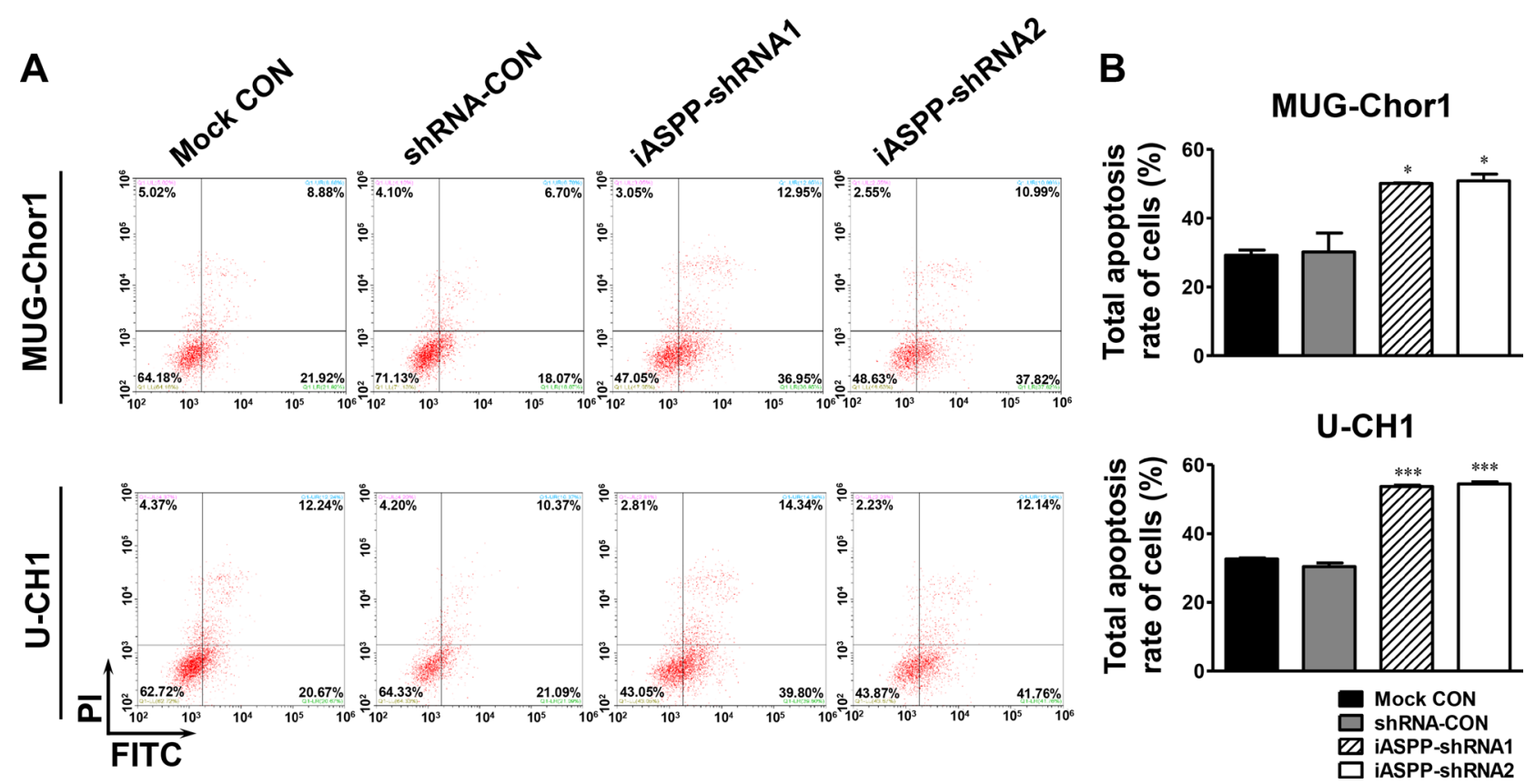

Figure 6: Effect of iASPP knockdown on cells apoptosis after cisplatin treatment. (A) Pictures of cells apoptosis detected by flow cytometry in $20 \mu \mathrm{M}$ cisplatin-treated MUG-Chor 1 and U-CH1 cells after the iASPP knockdown with iASPP-shRNAs. (B) The results of statistical analysis of total apoptosis rate in $20 \mu \mathrm{M}$ cisplatin-treated cells after the iASPP knockdown with iASPP-shRNAs. $n=3$. Mean \pm SEM. ${ }^{*} p<0.05,{ }^{* * *} p<0.001$, iASPP-shRNA1 group or iASPP-shRNA2 group $v s$. shRNA-CON group. CON: control. 
tumors and has a vital function in their occurrence and development [40, 41]. Cao et al. [23] demonstrated that the elevated iASPP in squamous cell cervical cancer correlated to patients' poor prognosis and chemo/ radiotherapy resistance. Kim et al. [42] showed that recurrent cases of oral cavity squamous cell carcinoma presented higher iASPP expression than primary cases, and these cases were associated with a lower survival rate. However, the function of iASPP in spinal chordoma and its clinical significance were previously unclear and explored in this study.

A total of 31 spinal chordomas were collected and tumor specimens were all confirmed pathologically to be conventional type by $\mathrm{HE}$ and IHC assays. For IHC assays, proteins including pan-cytokeratin, vimentin, epithelial membrane antigen (EMA), and S-100 were detected. These are considered as recognized biomarkers for the diagnosis of chordoma [43]. Brachyury, a novel tumorspecific biomarker for chordoma [44], was also assessed by IHC to confirm diagnosis. Subsequently, iASPP expression in chordoma tissues and two chordoma cell lines (MUG-Chor1 and U-CH1) were detected. Results demonstrated that iASPP predominantly expressed in the cytoplasm of chordoma cells and significantly overexpressed in chordoma tissues when compared to that in distant normal tissues and nucleus pulposus tissues. These results were consistent with the findings of iASPP expression in hepatocellular carcinoma and ovarian cancers that respectively reported by Lu et al. and Jiang et al. $[18,21]$. IHC staining showed that iASPP was highly expressed in $67.7 \%(21 / 31)$ of chordoma samples, but only $11.1 \%(2 / 18)$ of distant normal tissues, and $26.7 \%(4 / 15)$ of nucleus pulposus tissues; in addition, these differences were statistically significant. Likewise, iASPP expression in two chordoma cell lines observed by RT-qPCR and WB was also obviously higher than the normal control of primary nucleus pulposus cells. These results indicate that iASPP might be a crucial diagnostic biomarker for chordoma.

Clinically, it has showed that certain factors such as tumor size and location, local invasion, and surgical types and margins closely correlated to local recurrence and prognosis in chordoma patients $[6,45,46]$. Meanwhile, Morris et al. [47] reported that elevated iASPP was associated with tumor invasive growth, metastatic disease, and cancer-related mortality. They observed that iASPP was enriched in highly metastatic prostate cancer cells compared to expression in primary cells, and that iASPP-overexpressing cells were mostly distributed at the invasive leading edge. Lu et al. [18] also observed that iASPP overexpression in hepatocellular carcinoma was closely associated with tumor recurrence and patient survival time. Consistently, our data suggested that elevated iASPP expression was significantly associated with tumor invasion, but not with patient age, gender, or tumor size and location. In addition, 76.2\% (16/21) of patients presenting tumors with high iASPP expression developed recurrence, whereas recurrence occurred in only $20 \%(2 / 10)$ of patients having disease with low iASPP expression, and this difference was statistically significant.
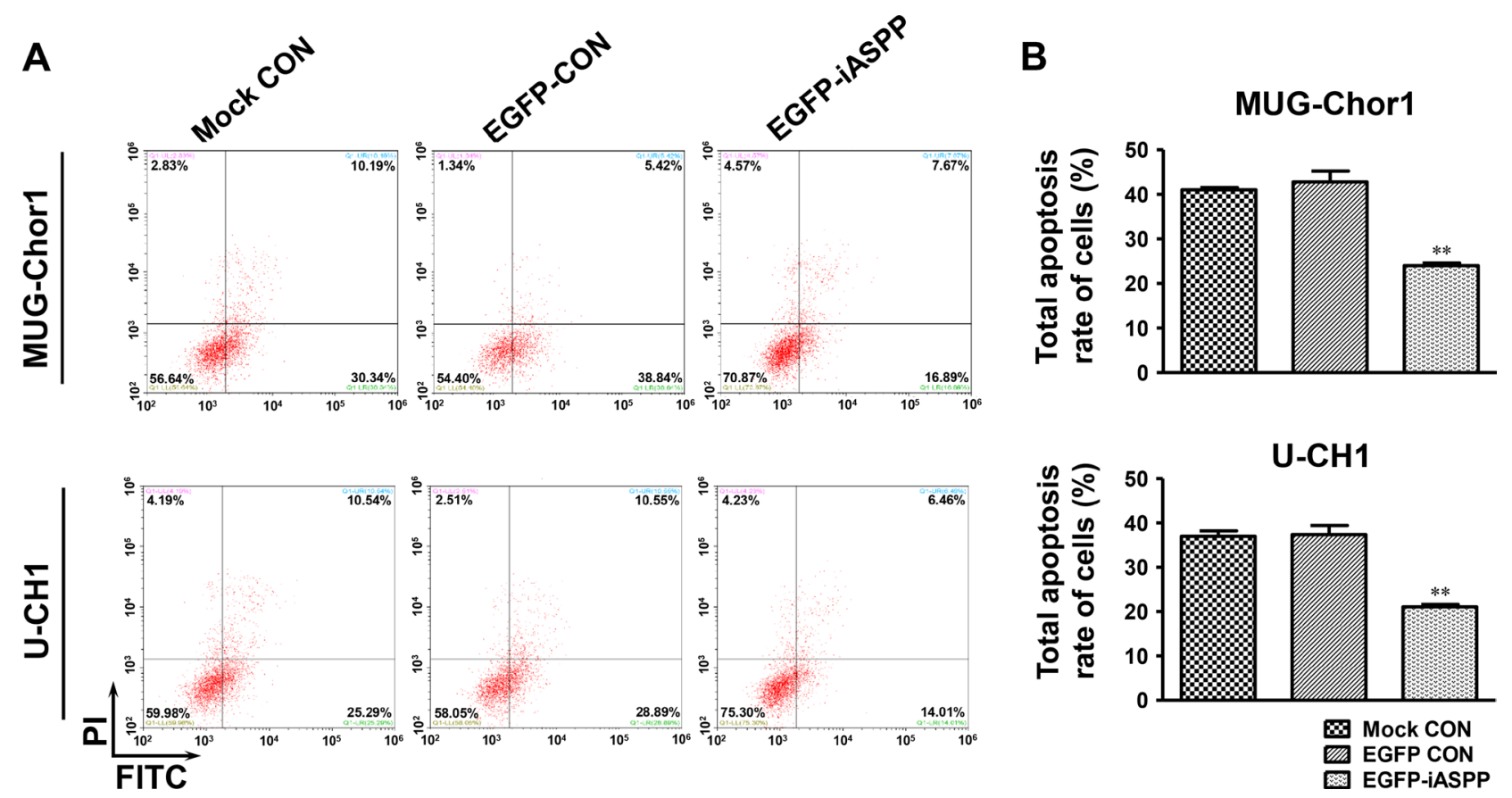

Figure 7: Effect of iASPP overexpression on cells apoptosis after cisplatin treatment. (A) Pictures of cells apoptosis detected by flow cytometry in $20 \mu \mathrm{M}$ cisplatin-treated MUG-Chor1 and U-CH1 cells after the iASPP overexpression with EGFP-iASPP. (B) The results of statistical analysis of total apoptosis rate in $20 \mu \mathrm{M}$ cisplatin-treated cells after the iASPP overexpression with EGFP-iASPP. $n=3$. Mean \pm SEM. $^{* *} p<0.01$, EGFP-iASPP group vs. EGFP-CON group. CON: control. 


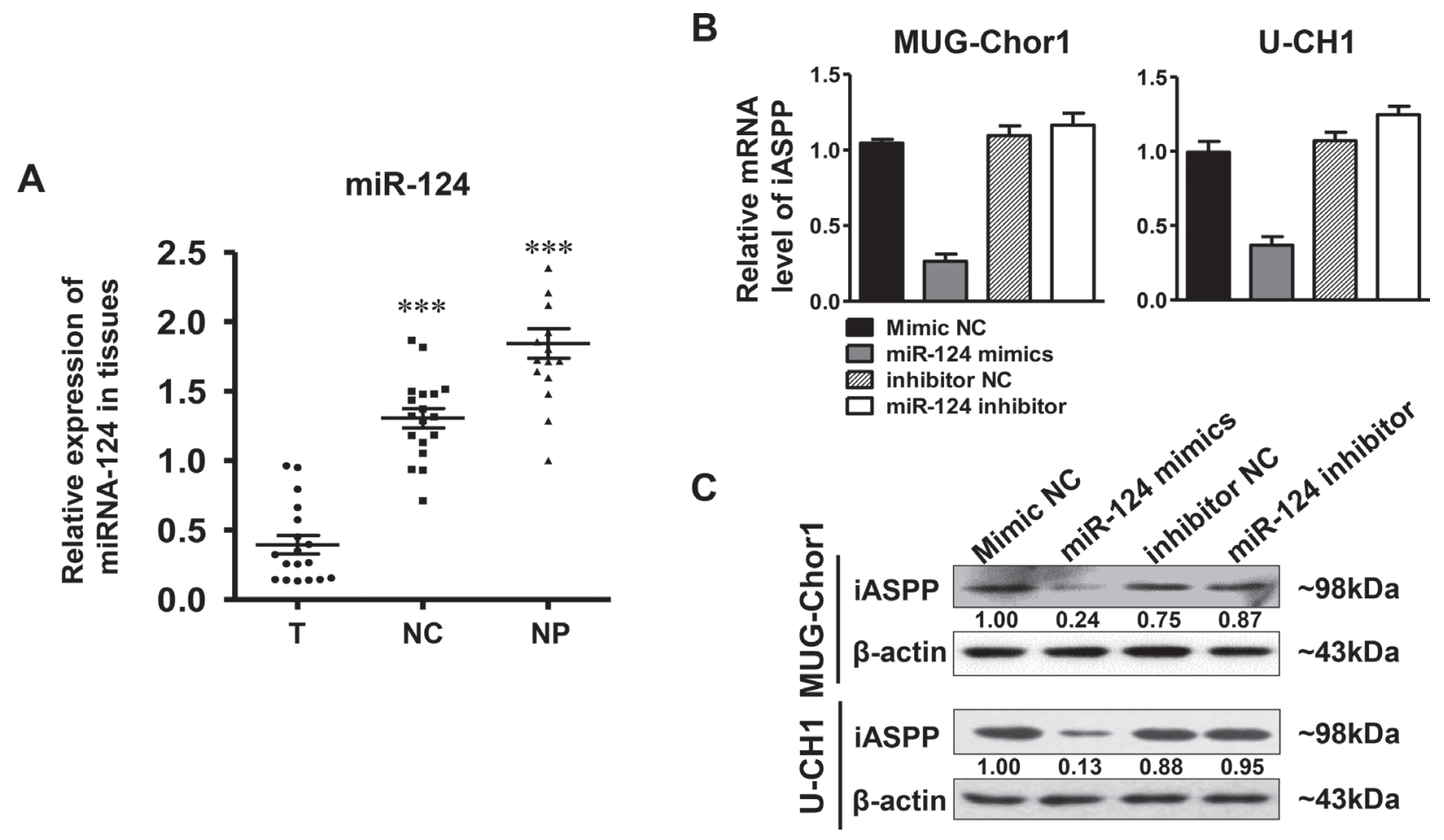

Figure 8: The expression of miRNA-124 in tissues and iASPP expression regulated by miRNA-124. (A) Relative expression of miRNA in tissues as detected by RT-qPCR. ${ }^{* * *} p<0.001 v$ s. tumor group. T: tumor group, NC: normal control tissues group, NP: nucleus pulposus tissues group. (B, C) Relative expression of iASPP in MUG-Chor1 and U-CH1 cells after the transfection of miRNA-124 mimics and inhibitor as detected by RT-qPCR (B) and WB (C). Numbers below picture C represent semi-quantitative analysis of each line by Quantity One software.

A

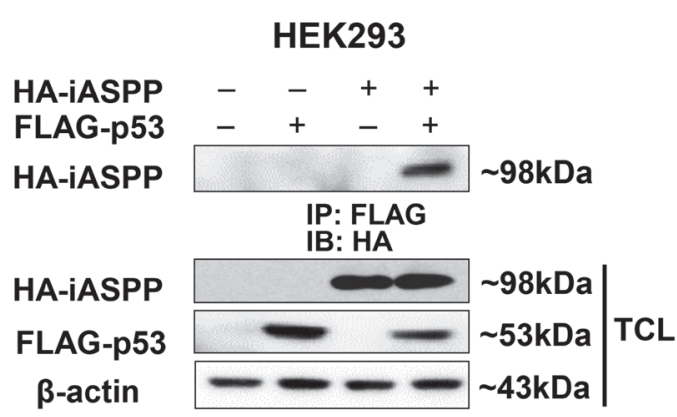

B

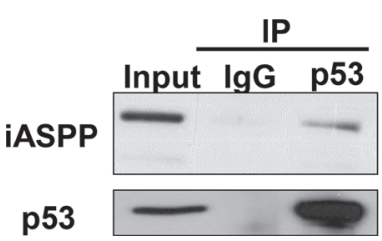

C

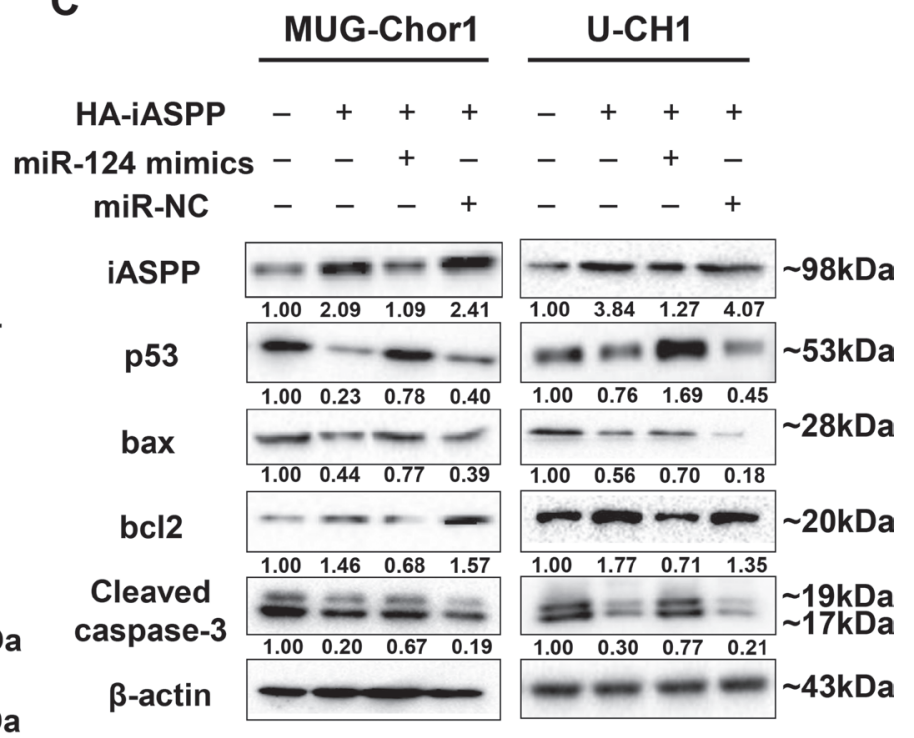

Figure 9: The interaction between iASPP and p53 detected by Co-Immunoprecipitation (Co-IP) assay and the regulation of miRNA-124 on apoptosis function of iASPP. (A, B) The Co-IP detection of the interaction between iASPP and p53 in exogenous (A) and endogenous conditions (B). (C) Changes of apoptosis-related proteins based on the regulation of miRNA-124 on iASPP in MUG-Chor1 and U-CH1 cells. Numbers below picture C represent semi-quantitative analysis of each line by Quantity One software. 
Furthermore, Kaplan-Meier survival plots and log-rank tests also suggested that the CDFS in patients with high iASPP expression was much shorter than in those with low iASPP expression. In addition, the cumulative survival rate of patients in the high iASPP expression group was notably lower than that in patients with the low expression group. Taken together, these results suggest that iASPP can serve as a novel prognostic factor and a predictor of tumor recurrence.

Many experimental studies have confirmed that the dysregulation of iASPP can notably disturbing the malignant biological behaviors of human cancers $[14,16,17]$. Chen et al. [16] suggested that the suppression of iASPP by employing shRNA technology led to an obvious inhibition of cellular proliferation in two non-small lung tumor cells harboring wild-type p53. Other studies also demonstrated that the proliferative and invasive properties of the pancreatic adenocarcinoma cells and the central nervous system lymphoma cells were remarkably weakened due to the downregulation of iASPP $[34,36]$. Similar to these reports, the cellular proliferation and invasion of chordoma cells in this study significantly decreased with iASPP silence, but enhanced with iASPP overexpression. In fact, iASPP overexpression in human malignant cells mainly relates to their chemotherapy resistance [14]. Jiang et al. [21] observed that ovarian cancer cells with iASPP overexpression conferred resistance to paclitaxel by reducing mitotic catastrophe via activation of separase, whereas knockdown of iASPP enhanced paclitaxel-mediated mitotic catastrophe through inactivating separase. Jia et al. [22] reported that the p53-dependent apoptosis of hematopoietic cells with iASPP overexpression reduced in response to cell damage stimuli (etoposide/VP-16), and the mutation accumulation was more common in these cells. Furthermore, the downregulation of endogenous iASPP caused a significant increase of p53-mediated apoptosis in human malignances induced by chemotherapeutics $[14,48]$. In this study, we also found that the apoptosis of chordoma cells notably increased with the knockdown of iASPP, but attenuated with iASPP overexpression. All these evidences imply that iASPP can be a potential therapeutic target for chordoma. miRNAs are a class of non-coding, singlestranded RNAs with a small size ( 22 nucleotides in length), which negatively modulate gene expression at post-transcriptional level by mainly binding to the 3 '-untranslated region (3'-UTR) of their target mRNAs [27]. Either the bioinformatics prediction of miRNA target based on sequence complementarity or the luciferase reporter assay used in previous studies both validated the binding and repressive effects of miRNA-124 on the 3'-UTR of iASPP [28]. The inversely regulation of miRNA-124 on iASPP expression has been confirmed to mediate the malignant progression including cellular proliferation, apoptosis and invasion in many tumor cells, such as glioblastoma, prostate cancer and colorectal cancer $[30,32,33]$. In this study, we observed that the level of miRNA-124 in chordoma tissues was significantly lower than the corresponding normal controls. What's more, there was a negative association between miRNA-124 and iASPP expression in chordoma cells validated by RT-qPCR and WB. These results suggested that iASPP overexpression was reversely regulated by miRNA-124, which was at a low level in chordoma.

The regulatory function on apoptosis by iASPP through p53 has been widely reported. Consistently, the results of co-immunoprecipitation assay confirmed that iASPP could interact with p53 in either exogenous or endogenous detection, which implied that the potential regulation of iASPP on the tumorigenesis of chordoma through p53. As a tumor suppressor, p53 exerts its function mainly by inducing cell cycle arrest or apoptosis, and p53dependent apoptosis typically follow the mitochondrial pathway. Activation of cytoplasmic p53 is sufficient to increase the transcription of various pro-apoptotic Bcl-2 family members, such as Bax, Noxa, and Puma; in the mitochondrion, p53 directly induces Bax oligomerization and forms inhibitory complexes with the anti-apoptotic Bcl-xl and Bcl-2, which causes the permeabilization of the mitochondrial membrane and cytochrome c release [49]. These changes subsequently activate caspase cascades, and apoptotic effector caspase- 3 is eventually activated to cause apoptosis [50]. The results showed that the level of p53 was significantly down-regulated by iASPP, followed by the up-regulation of anti-apoptotic protein bcl-2 and the down-regulation of pro-apoptotic proteins bax and caspase-3. However, the inhibition of iASPP by miRNA-124 mimics presented the reverse regulation effects on these proteins. These findings support that the overexpression of miRNA-124 can attenuate the inhibition of iASPP on p53, which potentially enhances the apoptosis of chordoma cells.

Besides, iASPP is considered as a substrate for caspases, which highlights its potential as a direct target that can be stimulated by apoptotic signals [51]. The signaling cascades of the Hedgehog/GLI-E2F1 axis or those downstream of hepatitis virus $\mathrm{X}$ protein-mediated $\mathrm{NF}-\kappa \mathrm{B}$ activation were also shown to modulate iASPP in tumor cells $[18,52]$. These data indicate that the causes of iASPP overexpression in tumors might be multifarious, and thus need to be explored intensively. Although iASPP regulates the biological behaviors in tumor cells mostly through p53-dependent pathways, recent studies also report its regulation in a p53-independent manner $[39,41,53]$. These evidences suggest that the downstream regulatory mechanisms of iASPP are diverse and complicated. Therefore, it is necessary to explore these issues in the context of chordoma in future.

In conclusion, iASPP overexpression is associated with the clinical outcome in spinal chordoma and influences the cellular proliferation, invasion, and their sensitivity to cisplatin. It might serve as a novel prognostic 
and predictive biomarker as well as a therapeutic target for chordoma. In addition, further study is necessary to explore the deeply regulatory mechanisms of iASPP in the tumorigenesis and progression of chordoma.

\section{MATERIALS AND METHODS}

\section{Patient data}

A total of 31 chordoma patients ( 22 men and 9 women) were included in the study, with an average age of 55.0 years (range 20 78 years). Lesions were located in the cervical vertebra in 19 patients and in the sacrococcygeal region in 12 patients. All patients received surgical treatment at the Department of Orthopedics, Peking University Third Hospital, from 2008 to 2015. Eighteen distant normal tissues obtained at least $3 \mathrm{~cm}$ from the surgical margins and 15 normal nucleus pulposus tissues derived from patients with lumbar disc herniation (LDH) or lumbar spinal stenosis (LSS) were collected as controls. The clinical information of chordoma patients was recorded in detail. The research was approved by the ethics committee of the Peking University Third Hospital (No. IRB00006761-2016048) and informed consent was obtained from all patients.

\section{IHC for iASPP}

The EnVision two-step staining technique was performed in IHC to detect iASPP expression in tissue samples as described previously [23]. Briefly, the tissue sections were dewaxed with xylene and a gradient concentration of ethanol, which was followed by antigen heat retrieval. The primary antibody was a rabbit antiiASPP polyclonal antibody (ab34898, 1:200, Abcam, Cambridge, MA, USA). Standard streptavidin-biotinperoxidase complex method and a biotinylated anti-IgG secondary antibody were applied. Samples were visualized with 3, 3'-diaminobenzidine (DAB). In addition, tissue sections were incubated with phosphate-buffered saline buffer (PBS) to replace the primary antibody as a negative control (Supplementary Figure 3A). Sections of human breast carcinoma with known positivity were used as the positive control (Supplementary Figure 3B). All sections were observed under one optical microscope (Leica, Frankfurt, Germany) and scored independently by two experienced pathologists who were blinded to the patient information.

The results of IHC staining were analyzed by combining the percentage of positive cells with the staining intensity as similar to previous report [16]. The percentage of positively stained cells was scored as 0 (none), 1 (1 25\%), 2 (26 50\%), 3 (51 75\%), or 4 (76 100\%). The color intensity was scored as 0 (negative), 1 (weak), 2 (moderate), and 3 (strong). The total score was calculated by the product of the staining intensity and the percentage of positive cells. Thus, the range of the final score was $0-12$. For statistical analyses, the expression of iASPP was divided into low expression (final score $\leq 6$ ) and high expression groups (final score $\geq 8$ ) according to the mean value of scores from all 31 data.

\section{Pathological diagnosis of chordoma samples}

The pathological diagnosis of tumor samples was assessed by $\mathrm{HE}$ and IHC staining. The primary antibodies used in IHC staining included mouse anti-pan cytokeratin monoclonal antibody (ab7753, 1:250, Abcam, Cambridge, MA, USA), rabbit anti-vimentin monoclonal antibody (ab92547, 1:250, Abcam), rabbit anti-S100 polyclonal antibody (BS1318, 1:100, Bioworld Technology, Inc. St. Louis Park, USA), mouse anti-EMA monoclonal antibody (ZM-0095, 1:100, ZSGB, Beijing, China), and rabbit antibrachyury polyclonal antibody (sc-20109, 1:250, Santa Cruz Biotechnology, Santa Cruz, CA, USA).

\section{Follow-up}

The imaging information for patients including the plain radiographs, computed tomography scans, and magnetic resonance images was collected. All patients were subjected to follow-up and the average follow-up period was 60.6 months (range 7 98 months). During follow-up, local recurrence was identified in 18 cases and the mean recurrence time was $20.1 \pm 14.8$ months. At the end of the follow-up, 11 patients experienced cancerrelated death. CDFS was defined as the time interval from resection of the first tumor to recurrence [54].

\section{Cell lines and cell culture}

Two human chordoma cell lines, MUG-Chor1 (CRL-3219) and U-CH1 (CRL-3217), purchased from the American Type Culture Collection (ATCC, Manassas, VA, USA) were not passaged for longer than 6 months during the study. They were cultured in Dulbecco's modified Eagle's medium (DMEM) and RPMI medium with a volume ratio of 4:1 (HyClone, Logan, UT, USA) which supplemented with $2 \mathrm{mM}$ L-glutamine (Gibco, Grand Island, NY, USA), 10\% fetal bovine serum (FBS) (HyClone), $100 \mathrm{U} / \mathrm{mL}$ penicillin, and $100 \mu \mathrm{g} / \mathrm{mL}$ streptomycin (Gibco). Cell culture flasks or dishes were pre-coated with rat tail collage type I (Corning Lasertron, Bedford, MA, USA). Human Prostatic Cancer (PC3) cells were cultured in DMEM medium supplemented with the components as similar to chordoma cells but without L-glutamine as an iASPP positive control. Human Emborynic Kidney (HEK293) cells were cultured as same as the culture condition of PC3 cells. All cells were maintained in an incubator containing $5 \% \mathrm{CO}_{2}$ at $37^{\circ} \mathrm{C}$. 


\section{Nucleus pulposus (NP) cell isolation and culture}

The primary cell culture method of NP was similar to others [55]. Briefly, human intervertebral disc NP tissues derived from the patients with LDH or LSS were rinsed and minced into small fragments of about $1 \mathrm{~mm}^{3}$. Then, the tissues were isolated by digestion with $0.25 \%$ trypsin (Invitrogen, Carlsbad, CA, USA) for about $20 \mathrm{~min}$ and $0.1 \%$ type II collagenase (Invitrogen) for $3 \sim 4 \mathrm{~h}$ at $37^{\circ} \mathrm{C}$. The digested mixture was collected and centrifuged at $1,500 \mathrm{rpm}$ for $10 \mathrm{~min}$. After carefully removed the supernatant, the remnant was re-suspended with DMEM-F12 culture medium and plated in the flasks. When the primary cells were firmly attached, the media were changed every 3 days.

\section{Lentivirus packaging and infection}

For knockdown of the endogenous iASPP gene (NM_001142502), shRNA targeting iASPP was cloned into the pLKD-CMV-U6 lentivirus vector (Obio Technology, Shanghai, China). Two effective shRNA target sequences were used as shRNA1 (5'-GCCTCAAAGGAGTAAAGTC-3') and shRNA2 (5'-ACTACTCTATCGTGGATTT-3'), and a scrambled plasmid (5'-TTCTCCGAACGTGTCACGT-3') was used as a negative control. The lentivirus expressing iASPPshRNA1, iASPP-shRNA2, and the control sequence named as pLKD-iASPP-shRNA1, pLKD-iASPP-shRNA2, and pLKD-shRNA-Control, respectively. Similarly, the full length CDS of the PPP1R13L gene (encoding iASPP) was cloned into the pLenti-EF1a-EGFP-P2A-Puro-CMVMCS-3Flag lentivirus vector, and the empty plasmid was used as a control. The lentivirus overexpressing iASPP and the corresponding control lentivirus named as pLentiEGFP-iASPP and pLenti-EGFP-Control, respectively.

\section{RT-qPCR assay}

Total RNA was extracted using TRIzol reagent (15596018, Invitrogen) and the cDNA of mRNA was synthesized from $1 \mu \mathrm{g}$ of total RNA with M-MLV (M170A, Promega, USA). RT-qPCR was performed using a commercial SYBR Green RT-PCR Kit (QPK-201, Takara, Otsu, Japan). The reverse transcription and detection of miRNA was performed using Bulge-Loop ${ }^{\mathrm{TM}}$ miRNA qRT-PCR Starter Kit (C10211, RiboBio Co., Guangzhou, China) and Bulge-Loop ${ }^{\mathrm{TM}}$ hsa-miR-124-5p qRT-PCR Primer Set (miRQ0004591-1-2, RiboBio Co., Guangzhou, China). The detetion of U6 snRNA was used as internal reference (MQP-0202, RiboBio Co., Guangzhou, China). The amplification parameters in this assay as follows: 1 cycle at $95^{\circ} \mathrm{C}$ for $10 \mathrm{~min}, 40$ cycles of $95^{\circ} \mathrm{C} 15 \mathrm{~s}$ and $60^{\circ} \mathrm{C}$ $1 \mathrm{~min}$. The specific primers of PPP1R13L and GAPDH were constructed by Sangon (Sangon Biotech, Shanghai, China) and their detailed sequences as follows: PPP1R13L (forward: 5'-CAGACAGCGAGCTATGAACG-3', reverse: 5'-GTGGCGCTAGTGAGGTTGTC-3'); GAPDH (forward: 5'-GGTGGTCTCCTCTGACTTCAACA-3', reverse: $\quad 5^{\prime}$-GTTGCTGTAGCCAAATTCGTTGC-3'). The mRNA and miRNA expression was respectively normalized to the expression of GAPDH and U6, and was expressed as $2^{-\Delta \Delta C t}$ after normalization. All samples were assayed in triplicate.

\section{WB assay}

Total protein was extracted using RIPA lysis buffer (Applygen Technologies Inc., Beijing, China). Protein concentration was determined using the BCA method and sample preparation was performed as described previously [56]. Equal amounts of protein were separated using an SDS-PAGE gel $(10 \%)$ and then transferred to a nitrocellulose membrane. Membranes were blocked with $5 \%$ BSA for $1 \mathrm{~h}$ and incubated with indicated primary antibodies as follows: anti-iASPP rabbit polyclonal antibody (ab34898, 1:1000, Abcam, Cambridge, MA, USA), anti-p53 rabbit monoclonal antibody (2527, 1:1000, Cell signaling technology, CST, Danvers, MA, USA), anti-bax rabbit polyclonal antibody (2772, 1:1000, CST ), anti-bcl-2 rabbit polyclonal antibody (2872, 1:1000, CST), anti-caspase-3 rabbit polyclonal antibody (9662, 1:1000, CST), anti-FLAG rabbit polyclonal antibody (F7425, 1:2000, Sigma-Aldrich, St. Louis, MO, USA), and anti-HA TAG mouse monoclonal antibody (SAB1305536, 1:1000, Sigma-Aldrich). Anti- $\beta$-actin mouse monoclonal antibody (CW0096M, 1:3000, CWBIO, Beijing, China) was used as the internal control. IRDye $800 \mathrm{CW}$-conjugated goat (polyclonal) anti-rabbit/mouse IgG antibody (1:10000, LI-COR ${ }^{\circledR}$ Biosciences, Lincoln, NE, USA) was used as the secondary antibody. The bands were visualized using an Odyssey $\mathrm{CLx}$ infrared imaging system (LI-COR ${ }^{\circledR}$ Biosciences) and its relative gray value was measured using by Quantity One software (Bio-Rad, Hercules, CA, USA).

\section{Co-Immunoprecipitation (Co-IP) assay}

Plasmids encoding iASPP and $\mathrm{p} 53$ were constructed by digested PCR products and linked into the pCMV-HA (PT3283-5, Clontech, Takara Bio, USA) and pFLAGCMV2 vectors (E7398, Sigma-Aldrich). HEK293 cells were inoculated into $10-\mathrm{cm}$ dishes at a density of $2.0 \times 10^{7}$ and then transfected with $8.0 \mu \mathrm{g}$ pCMV-iASPPHA plasmid combined with $7.0 \mu \mathrm{g}$ pFLAG-p53 plasmid together at $80 \%$ confluence using lipofectamine 2000 reagent (Invitrogen) according to the protocol. Cells transfected with single plasmid or empty control plasmid was used as controls. At $48 \mathrm{~h}$ post-transfection, cells were collected for protein extraction with lysis buffer $(100 \mathrm{mM}$ $\mathrm{NaCl}, 2.5 \mathrm{mM} \mathrm{MgCl}, 0.1 \mathrm{mM}$ EDTA and $0.01 \%$ Triton $\mathrm{X}-100, \mathrm{pH}$ 8.0). An aliquot (1\%) of lysis was isolated for WB analysis to detect the expression of iASPP and p53. 
Then, the remaining supernatants were incubated with FLAG-M2 beads (A2220, Sigma-Aldrich) at for $12 \mathrm{~h}$ at $4^{\circ} \mathrm{C}$. Beads were washed three times with lysis buffer. The co-precipitated proteins were eluted and analyzed by WB with anti-HA monoclonal antibody.

To explore the endogenous interaction between iASPP and p53, MUG-Chor1 and U-CH1 cells were collected and lysis for protein extraction. The supernatant of cell lysates were collected and dithiobis (succinimidyl propionate) (DSP) (22585, Thermo Fisher Scientific, Waltham, MA, USA) was added into supernatant with $1 \mathrm{mg} / \mathrm{mL}$ for $2 \mathrm{~h}$ at $4{ }^{\circ} \mathrm{C}$. An aliquot $(1 \%)$ of lysis was isolated for WB analysis to detect the expression of iASPP and $\mathrm{p} 53$. Then, the supernatant was immunoprecipitated overnight at $4^{\circ} \mathrm{C}$ with anti-p53 monoclonal antibody. The resultant Mab-protein complexes were immunoprecipitated with protein A/G agarose (sc-500775, Santa Cruz Biotechnology) for $2 \mathrm{~h}$ at $4^{\circ} \mathrm{C}$. Then the agarose were washed three times with wash buffer $(50$ $\mathrm{mM} \mathrm{NaCl}, 2.5 \mathrm{mM} \mathrm{MgCl}_{2}, 0.1 \mathrm{mM}$ EDTA and $0.01 \%$ Triton $\mathrm{X}-100, \mathrm{pH} 8.0$ ). The precipitates were then detected by WB with anti-iASPP antibody.

\section{Oligonucleotide transfection}

MUG-Chor1 and U-CH1 cells were inoculated into 6-well plates at a density of $5.0 \times 10^{5}$ per well and were transiently transfected with miRNA-124 mimics (miR10004591-1-5), miRNA-124 inhibitor (miR200045911-5), and relevant controls including micrON ${ }^{\mathrm{TM}}$ mimic Negative Control (miR01101-1-5) and micrOFFTM inhibitor Negative Control (miR02101-1-5) that purchased from RiboBio Co., Guangzhou, China. Lipofectamine RNAi MAX (Invitrogen) was used in transfection when cells reached $50 \%$ confluence. At $72 \mathrm{~h}$ post-transfection, cells were collected for the extraction of RNA and proteins, respectively. The effect of miRNA-124 on iASPP expression was determined by RT-qPCR and WB.

\section{CCK-8 cell proliferation assay}

Cells were seeded in 96-well dishes at a final density of $5 \times 10^{3}$ (MUG-Chor 1$)$ or $8 \times 10^{3}(\mathrm{U}-\mathrm{CH} 1)$ cells per well and incubated for 0,4 , and $7 \mathrm{~d}$. At each time point, CCK-8 reagent (Dojindo Laboratories, Kumamoto, Japan) was added and incubated for $3 \mathrm{~h}$ according to the manufacturer's protocol. The mean absorbance at $450 \mathrm{~nm}$ from six wells was calculated. The experiment was repeated three times.

\section{Colony formation assay}

Cells were seeded into 12 -well plates (300 cells per well) and incubated for 2 weeks, and then fixed with paraformaldehyde and stained with crystal violet. The number of colonies in each well was counted under an inverted microscope (Leica, Frankfurt, Germany) and only more than 50 cells could be regarded as one colony. Three replicate wells were used for each condition, and the experiment was repeated three times.

\section{Annexin V-FITC/PI apoptosis assay}

The apoptosis of cells that treated with $20 \mu \mathrm{M}$ of cisplatin for $48 \mathrm{~h}$ was determined by flow cytometry using an Annexin V-FITC/PI apoptosis detection kit (BioVision Co, Milpitas, CA, USA). Briefly, $1 \times 10^{6}$ cells were resuspended in $300 \mu \mathrm{L}$ of $1 \times$ binding buffer. Then, $5 \mu \mathrm{L}$ of annexin V-FITC and $5 \mu \mathrm{L}$ of PI reagent were added and samples were incubated for $10 \mathrm{~min}$ in the dark. The intensity analysis of fluorescence-activated cells was performed by flow cytometry (BD Biosciences, San Jose, CA, USA). All results were repeated for three times.

\section{Transwell invasion assay}

A total of $5 \times 10^{3}$ cells, re-suspended in $50 \mu \mathrm{L}$ of serum-free medium, was added to the upper chamber, which was pre-coated with Matrigel (BD Biosciences, Franklin Lakes, NJ, USA) for the invasion assay. The lower chamber was filled with $600 \mu \mathrm{L}$ of culture medium containing $10 \% \mathrm{FBS}$. After incubation for $36 \mathrm{~h}$ at $37^{\circ} \mathrm{C}$, invading cells, on the lower surface of the filter membrane, were fixed by paraformaldehyde and stained with crystal violet. Under an inverted microscope, five random fields were selected to count under high magnification $(200 \times)$. Each experiment was repeated three times.

\section{Statistical analysis}

Data were analyzed using IBM SPSS statistics 20.0 software. A Pearson's chi-squared test was used to explore the association between iASPP expression and clinical data from patients. The Kaplan-Meier survival curve and log-rank test were applied to evaluate the correlation between iASPP expression and CDFS and cumulative survival rate in chordoma patients by univariate analysis. For comparison with the control group, a one-way analysis of variance (ANOVA) with a post hoc Dunnett analysis was performed. On statistical analysis, the data were analyzed based on the mean \pm standard deviation (SD) and their presentations in figures were the mean \pm standard error of the mean (SEM). The statistical significance was defined as a value of $p<0.05$ based on two-tailed tests.

\section{Abbreviations}

ASPP: apoptosis stimulating protein of p53, NF$\kappa \mathrm{B}$ : nuclear factor kappa B, RAI: Rel-associated inhibitor, NP: nucleus pulposus, CDS: coding domain sequence, HE: hematoxylin and eosin, IHC: immunohistochemistry, 
RT-qPCR: reverse transcription quantitative polymerase chain reaction, WB: Western blotting, CDFS: continuous disease-free survival time, EMA: epithelial membrane antigen, DAB: 3,3'-diaminobenzidine, PBS: phosphatebuffered saline buffer, ATCC: American Type Culture Collection, DMEM: Dulbecco's modified Eagle's medium, FBS: fetal bovine serum, Co-IP: Co-Immunoprecipitation, SEM: standard error of the mean, ANOVA: one-way analysis of variance

\section{Author contributions}

XGL, YLM and BZ conceived and designed the study; XGL, ZJL, HZ, LJ, FW, MY and FLW participated in the acquisition of samples and clinical data; YLM, BZ and NFX were responsible for literature research; XL, LY, YQW and PW involved in statistical analysis and interpretation of data; YLM drafted the manuscript; CL and GPH carried out the supplementary experiments. XGL and ZJL revised the manuscript. All authors read and approved the final manuscript.

\section{ACKNOWLEDGMENTS}

We would like to thank the Medical Research Center of Peking University Third Hospital for technical guidance. PC3 and HEK293 cells were kindly donated by Wei Zhang, PhD from the Institute of Zoology, Chinese Academy of Science.

\section{CONFLICTS OF INTEREST}

The authors declare no competing interests.

\section{FUNDING}

This study was supported by the National Natural Science Foundation of China (No. 81472041; No. 81641103) and the Specialized Research Fund for the Doctoral Program of Higher Education of China (No. 20130001120091).

\section{REFERENCES}

1. Smoll NR, Gautschi OP, Radovanovic I, Schaller K, Weber DC. Incidence and relative survival of chordomas: the standardized mortality ratio and the impact of chordomas on a population. Cancer. 2013; 119:2029-2037.

2. Jemal A, Siegel R, Ward E, Murray T, Xu J, Thun MJ. Cancer statistics, 2007. CA Cancer J Clin. 2007; 57:43-66.

3. McMaster ML, Goldstein AM, Bromley CM, Ishibe N, Parry DM. Chordoma: incidence and survival patterns in the United States, 1973-1995. Cancer Causes Control. 2001; 12:1-11.

4. Moojen WA, Vleggeert-Lankamp CL, Krol AD, Dijkstra SP. Long-term results: adjuvant radiotherapy in en bloc resection of sacrococcygeal chordoma is advisable. Spine (Phila Pa 1976). 2011; 36:E656-661.

5. Fleming GF, Heimann PS, Stephens JK, Simon MA, Ferguson MK, Benjamin RS, Samuels BL. Dedifferentiated chordoma. Response to aggressive chemotherapy in two cases. Cancer. 1993; 72:714-718.

6. Ruggieri P, Angelini A, Ussia G, Montalti M, Mercuri M. Surgical margins and local control in resection of sacral chordomas. Clin Orthop Relat Res. 2010; 468:2939-2947.

7. Hanna SA, Aston WJ, Briggs TW, Cannon SR, Saifuddin A. Sacral chordoma: can local recurrence after sacrectomy be predicted? Clin Orthop Relat Res. 2008; 466:2217-2223.

8. Rotondo RL, Folkert W, Liebsch NJ, Chen YL, Pedlow FX, Schwab JH, Rosenberg AE, Nielsen GP, Szymonifka J, Ferreira AE, Hornicek FJ, DeLaney TF. High-dose protonbased radiation therapy in the management of spine chordomas: outcomes and clinicopathological prognostic factors. J Neurosurg Spine. 2015; 23:788-797.

9. Bowyer J, Natha S, Marsh I, Foy P. Visual complications of proton beam therapy for clival chordoma. Eye (Lond). $2003 ; 17: 318-323$.

10. Vousden $\mathrm{KH}, \mathrm{Lu} \mathrm{X}$. Live or let die: the cell's response to p53. Nat Rev Cancer. 2002; 2:594-604.

11. Naka T, Boltze C, Kuester D, Schulz TO, Schneider-Stock R, Kellner A, Samii A, Herold C, Ostertag H, Roessner A. Alterations of G1-S checkpoint in chordoma: the prognostic impact of p53 overexpression. Cancer. 2005; 104:1255-1263.

12. Horbinski C, Oakley GJ, Cieply K, Mantha GS, Nikiforova MN, Dacic S, Seethala RR. The prognostic value of Ki-67, p53, epidermal growth factor receptor, 1p36, 9p21, 10q23, and 17p13 in skull base chordomas. Arch Pathol Lab Med. 2010; 134:1170-1176.

13. Trigiante G, Lu X. ASPP [corrected] and cancer. Nat Rev Cancer. 2006; 6:217-226.

14. Bergamaschi D, Samuels Y, O'Neil NJ, Trigiante G, Crook T, Hsieh JK, O’Connor DJ, Zhong S, Campargue I, Tomlinson ML, Kuwabara PE, Lu X. iASPP oncoprotein is a key inhibitor of p53 conserved from worm to human. Nat Genet. 2003; 33:162-167.

15. Samuels-Lev Y, O'Connor DJ, Bergamaschi D, Trigiante G, Hsieh JK, Zhong S, Campargue I, Naumovski L, Crook T, Lu X. ASPP proteins specifically stimulate the apoptotic function of p53. Mol Cell. 2001; 8:781-794.

16. Chen J, Xie F, Zhang L, Jiang WG. iASPP is over-expressed in human non-small cell lung cancer and regulates the proliferation of lung cancer cells through a p53 associated pathway. BMC Cancer. 2010; 10:694.

17. Wang C, Gao C, Chen Y, Yin J, Wang P, Lv X. Expression pattern of the apoptosis-stimulating protein of p53 family in p53+ human breast cancer cell lines. Cancer Cell Int. 2013; 13:116.

18. Lu B, Guo H, Zhao J, Wang C, Wu G, Pang M, Tong X, Bu F, Liang A, Hou S, Fan X, Dai J, Wang H, et al. Increased expression of iASPP, regulated by hepatitis $B$ virus $X$ 
protein-mediated NF-kappaB activation, in hepatocellular carcinoma. Gastroenterology. 2010; 139:2183-2194 e2185.

19. Kong F, Shi X, Li H, Li P, Yu J, Li X, Chen J, Sun Y, Jia Y. Increased expression of iASPP correlates with poor prognosis in FIGO IA2-IIA cervical adenocarcinoma following a curative resection. Am J Cancer Res. 2015; 5:1217-1224.

20. Zhang X, Wang M, Zhou C, Chen S, Wang J. The expression of iASPP in acute leukemias. Leuk Res. 2005; 29:179-183.

21. Jiang L, Siu MK, Wong OG, Tam KF, Lu X, Lam EW, Ngan HY, Le XF, Wong ES, Monteiro LJ, Chan HY, Cheung AN. iASPP and chemoresistance in ovarian cancers: effects on paclitaxel-mediated mitotic catastrophe. Clin Cancer Res. 2011; 17:6924-6933.

22. Jia Y, Peng L, Rao Q, Xing H, Huai L, Yu P, Chen Y, Wang C, Wang M, Mi Y, Wang J. Oncogene iASPP enhances self-renewal of hematopoietic stem cells and facilitates their resistance to chemotherapy and irradiation. FASEB J. 2014; 28:2816-2827.

23. Cao L, Huang Q, He J, Lu J, Xiong Y. Elevated expression of iASPP correlates with poor prognosis and chemoresistance/radioresistance in FIGO Ib1-IIa squamous cell cervical cancer. Cell Tissue Res. 2013; 352:361-369.

24. Bell HS, Ryan KM. iASPP inhibition: increased options in targeting the p53 family for cancer therapy. Cancer Res. 2008; 68:4959-4962.

25. Liu ZJ, Cai Y, Hou L, Gao X, Xin HM, Lu X, Zhong S, $\mathrm{Gu}$ SZ, Chen J. Effect of RNA interference of iASPP on the apoptosis in MCF-7 breast cancer cells. Cancer Invest. 2008; 26:878-882.

26. Hwang HW, Mendell JT. MicroRNAs in cell proliferation, cell death, and tumorigenesis. Br J Cancer. 2007; 96:R40-44.

27. Esquela-Kerscher A, Slack FJ. Oncomirs - microRNAs with a role in cancer. Nat Rev Cancer. 2006; 6:259-269.

28. Liu X, Li F, Zhao S, Luo Y, Kang J, Zhao H, Yan F, Li S, Ji X. MicroRNA-124-mediated regulation of inhibitory member of apoptosis-stimulating protein of p53 family in experimental stroke. Stroke. 2013; 44:1973-1980.

29. Yi H, Huang Y, Yang F, Liu W, He S, Hu X. MicroRNA-182 aggravates cerebral ischemia injury by targeting inhibitory member of the ASPP family (iASPP). Arch Biochem Biophys. 2017; 620:52-58.

30. Chen J, Xiao H, Huang Z, Hu Z, Qi T, Zhang B, Tao X, Liu SH. MicroRNA124 regulate cell growth of prostate cancer cells by targeting iASPP. Int J Clin Exp Pathol. 2014; 7:2283-2290.

31. Liu K, Yao H, Lei S, Xiong L, Qi H, Qian K, Liu J, Wang P, Zhao H. The miR-124-p63 feedback loop modulates colorectal cancer growth. Oncotarget. 2017; 8:29101-29115. http://doi.org/10.18632/oncotarget.16248.

32. Liu K, Zhao H, Yao H, Lei S, Lei Z, Li T, Qi H. MicroRNA-124 regulates the proliferation of colorectal cancer cells by targeting iASPP. Biomed Res Int. 2013; 2013:867537.

33. Zhao WH, Wu SQ, Zhang YD. Downregulation of miR-124 promotes the growth and invasiveness of glioblastoma cells involving upregulation of PPP1R13L. Int J Mol Med. 2013; 32:101-107.

34. Liang S, Gong X, Zhang G, Huang G, Lu Y, Li Y. MicroRNA-140 regulates cell growth and invasion in pancreatic duct adenocarcinoma by targeting iASPP. Acta Biochim Biophys Sin (Shanghai). 2016; 48:174-181.

35. Zhao H, Peng R, Liu Q, Liu D, Du P, Yuan J, Peng G, Liao Y. The lncRNA H19 interacts with miR-140 to modulate glioma growth by targeting iASPP. Arch Biochem Biophys. 2016; 610:1-7.

36. Liang XG, Meng WT, Hu LJ, Li L, Xing H, Xie G, Wang AQ, Jia YQ. MicroRNA-184 Modulates Human Central Nervous System Lymphoma Cells Growth and Invasion by Targeting iASPP. J Cell Biochem. 2017; 118: 2645-2653.

37. Lei $\mathrm{R}$, Xue $\mathrm{M}$, Zhang L, Lin Z. Long noncoding RNA MALAT1-regulated microRNA 506 modulates ovarian cancer growth by targeting iASPP. Onco Targets Ther. 2017; 10:35-46.

38. Yakkioui Y, van Overbeeke JJ, Santegoeds R, van Engeland M, Temel Y. Chordoma: the entity. Biochim Biophys Acta. 2014; 1846:655-669.

39. Cai Y, Qiu S, Gao X, Gu SZ, Liu ZJ. iASPP inhibits p53independent apoptosis by inhibiting transcriptional activity of $\mathrm{p} 63 / \mathrm{p} 73$ on promoters of proapoptotic genes. Apoptosis. 2012; 17:777-783.

40. Deng Q, Sheng L, Su D, Zhang L, Liu P, Lu K, Ma S. Genetic polymorphisms in ATM, ERCC1, APE1 and iASPP genes and lung cancer risk in a population of southeast China. Med Oncol. 2011; 28:667-672.

41. Zhang B, Xiao HJ, Chen J, Tao X, Cai LH. Inhibitory member of the apoptosis-stimulating protein of p53 (ASPP) family promotes growth and tumorigenesis in human $\mathrm{p} 53$ deficient prostate cancer cells. Prostate Cancer Prostatic Dis. 2011; 14:219-224.

42. Kim JW, Roh JL, Park Y, Cho KJ, Choi SH, Nam SY, Kim SY. Cytoplasmic iASPP expression as a novel prognostic indicator in oral cavity squamous cell carcinoma. Ann Surg Oncol. 2015; 22:662-669.

43. Rosenberg AE, Brown GA, Bhan AK, Lee JM. Chondroid chordoma - a variant of chordoma. A morphologic and immunohistochemical study. Am J Clin Pathol. 1994; 101:36-41.

44. Vujovic S, Henderson S, Presneau N, Odell E, Jacques TS, Tirabosco R, Boshoff C, Flanagan AM. Brachyury, a crucial regulator of notochordal development, is a novel biomarker for chordomas. J Pathol. 2006; 209:157-165.

45. Bergh P, Kindblom LG, Gunterberg B, Remotti F, Ryd W, Meis-Kindblom JM. Prognostic factors in chordoma of the sacrum and mobile spine: a study of 39 patients. Cancer. 2000; 88:2122-2134.

46. Schwab JH, Healey JH, Rose P, Casas-Ganem J, Boland PJ. The surgical management of sacral chordomas. Spine (Phila Pa 1976). 2009; 34:2700-2704.

47. Morris EV, Cerundolo L, Lu M, Verrill C, Fritzsche F, White MJ, Thalmann GN, ten Donkelaar CS, Ratnayaka I, 
Salter V, Hamdy FC, Lu X, Bryant RJ. Nuclear iASPP may facilitate prostate cancer progression. Cell Death Dis. 2014; 5:e1492.

48. Liu H, Wang M, Diao S, Rao Q, Zhang X, Xing H, Wang J. siRNA-mediated down-regulation of iASPP promotes apoptosis induced by etoposide and daunorubicin in leukemia cells expressing wild-type p53. Leuk Res. 2009; 33:1243-1248.

49. Amaral JD, Xavier JM, Steer CJ, Rodrigues CM. The role of p53 in apoptosis. Discov Med. 2010; 9:145-152.

50. Ma Y, Zhu B, Yong L, Song C, Liu X, Yu H, Wang P, Liu Z, Liu X. Regulation of Intrinsic and Extrinsic Apoptotic Pathways in Osteosarcoma Cells Following Oleandrin Treatment. Int J Mol Sci. 2016; 17.

51. Hu Y, Ge W, Wang X, Sutendra G, Zhao K, Dedeic Z, Slee EA, Baer C, Lu X. Caspase cleavage of iASPP potentiates its ability to inhibit p53 and NF-kappaB. Oncotarget. 2015; 6:42478-42490. http://doi.org/10.18632/ oncotarget.6478.

52. Pandolfi S, Montagnani V, Lapucci A, Stecca B. HEDGEHOG/GLI-E2F1 axis modulates iASPP expression and function and regulates melanoma cell growth. Cell Death Differ. 2015; 22:2006-2019.
53. Chikh A, Matin RN, Senatore V, Hufbauer M, Lavery D, Raimondi C, Ostano P, Mello-Grand M, Ghimenti C, Bahta A, Khalaf S, Akgul B, Braun KM, et al. iASPP/p63 autoregulatory feedback loop is required for the homeostasis of stratified epithelia. EMBO J. 2011; 30:4261-4273.

54. Zhou M, Chen K, Yang H, Wang G, Lu J, Ji Y, Wu C, Chen C. Expression of insulin-like growth factor II mRNAbinding protein 3 (IMP3) in sacral chordoma. J Neurooncol. 2014; 116:77-82.

55. Zhu Y, Liang $\mathrm{Y}$, Zhu $\mathrm{H}$, Lian $\mathrm{C}$, Wang L, Wang $\mathrm{Y}$, $\mathrm{Gu} \mathrm{H}$, Zhou G, Yu X. The generation and functional characterization of induced pluripotent stem cells from human intervertebral disc nucleus pulposus cells. Oncotarget. 2017; 8:42700-42711. http://doi.org/10.18632/ oncotarget. 17446.

56. Ma Y, Zhu B, Liu X, Yu H, Yong L, Liu X, Shao J, Liu Z. Inhibition of oleandrin on the proliferation show and invasion of osteosarcoma cells in vitro by suppressing Wnt/ beta-catenin signaling pathway. J Exp Clin Cancer Res. $2015 ; 34: 115$. 